\title{
Componentwise bounds for nearly completely decomposable Markov chains using stochastic comparison and reordering is
}

\author{
Nihal Pekergin ${ }^{\mathrm{a}, \mathrm{b}}$, Tuğrul Dayar ${ }^{\mathrm{c}, *}$, Denizhan N. Alparslan ${ }^{\mathrm{d}}$ \\ ${ }^{a}$ PRiSM, Université de Versailles-St.Quentin, 45 av. des Etats Unis, Versailles 78035, France \\ ${ }^{\mathrm{b}}$ CERMSEM, Université de Paris I, 106-112 Bld de l'Hopital, Paris 75647, France \\ ${ }^{\mathrm{c}}$ Department of Computer Engineering, Bilkent University, Eng. Fac Bldg RM EA521, 06800 Bilkent, Ankara, Turkey \\ d Computer Science Telecommunications, University of Missouri, 454 Robert H. Flarsheim Hall, 5100 Rockhill Road, Kansas City, \\ MO 64110, USA
}

Received 1 August 2000; accepted 3 March 2004

Available online 13 May 2004

\begin{abstract}
This paper presents an improved version of a componentwise bounding algorithm for the state probability vector of nearly completely decomposable Markov chains, and on an application it provides the first numerical results with the type of algorithm discussed. The given two-level algorithm uses aggregation and stochastic comparison with the strong stochastic (st) order. In order to improve accuracy, it employs reordering of states and a better componentwise probability bounding algorithm given st upper- and lower-bounding probability vectors. Results in sparse storage show that there are cases in which the given algorithm proves to be useful.
\end{abstract}

(C) 2004 Elsevier B.V. All rights reserved.

Keywords: Markov processes; Near complete decomposability; Stochastic comparison; Reorderings; Aggregation

\section{Introduction}

Nearly completely decomposable (NCD) Markov chains (MCs) [2,12,19] are irreducible stochastic matrices that can be symmetrically permuted [4] to the block form

\footnotetext{
This work is supported by TÜBITAK-CNRS joint grant.

${ }^{*}$ Corresponding author. Tel.: +90-312-290-1981; fax: +90-312-266-4047.

E-mail addresses: nihal.pekergin@prism.uvsq.fr (N. Pekergin), tugrul@cs.bilkent.edu.tr (T. Dayar), dna5a0@umkc.edu (D.N. Alparslan).
} 


$$
P_{n \times n}=\left(\begin{array}{cccc}
P_{1,1} & P_{1,2} & \cdots & P_{1, N} \\
P_{2,1} & P_{2,2} & \cdots & P_{2, N} \\
\vdots & \vdots & \ddots & \vdots \\
P_{N, 1} & P_{N, 2} & \cdots & P_{N, N}
\end{array}\right) n_{2}
$$

in which nonzero elements of the off-diagonal blocks are small compared with those of the diagonal blocks $\left[19\right.$, p. 286]. Let $P=\operatorname{diag}\left(P_{1,1}, P_{2,2}, \ldots, P_{N, N}\right)+F$. The diagonal blocks $P_{i, i}$ are square, of order $n_{i}$, with $n=\sum_{i=1}^{N} n_{i}$. The quantity $\|F\|_{\infty}$ is referred to as the degree of coupling and is taken to be a measure of the decomposability of $P$. When the chain is NCD, it has eigenvalues close to 1 , and the poor separation of the unit eigenvalue implies a slow rate of convergence for standard matrix iterative methods [7, p. 290]. Hence, NCD Markov chains are said to be ill-conditioned [12, p. 258]. On the other hand, if $P$ were reducible, we would decompose the chain into its irreducible (i.e., isolated) and transient subclasses of states as in Eq. (1.20) of $[19$, p. 26] and continue our analysis on the irreducible subclasses.

Such matrices arise in queuing network analysis, large-scale economic modeling, and computer systems performance evaluation. The measures of interest for these systems may be obtained either from the longrun distribution of state probabilities by solving a homogeneous system of linear equations with a singular coefficient matrix under a normalization constraint (i.e., steady state analysis), or from the state probability distribution at a particular time instant by solving a set of first order ordinary differential equations using an initial state probability distribution (i.e., transient analysis).

To each NCD MC corresponds an irreducible coupling matrix [12], $C$, whose $(i, j)$ th element is given by

$$
c_{i, j}=\frac{\pi_{i}}{\left\|\pi_{i}\right\|_{1}} P_{i, j} e \quad \forall i, j \in\{1,2, \ldots, N\} .
$$

Here $e$ represents a column vector of all ones and the steady state probability (row) vector $\pi$ is partitioned conformally with $P$ in Eq. (1) such that $\pi=\left(\pi_{1}, \pi_{2}, \ldots, \pi_{N}\right)$, where each $\pi_{i}$ is a row vector having $n_{i}$ elements. The coupling matrix shows the evolution of the system when each NCD partition is treated as a single aggregated state. In other words, $C$ describes the coupling among NCD partitions, and its steady state vector gives the steady state probability of being in each NCD partition. Note that one needs to know $\pi$ to compute $C$ exactly.

For the partitioning in Eq. (1), the stochastic complement [12] of $P_{i, i}$ for $i \in\{1,2, \ldots, N\}$ is given by

$$
\bar{P}_{i, i}=P_{i, i}+P_{i,:}\left(I-P_{i}\right)^{-1} P_{:, i},
$$

where $P_{i,:}$ is the $n_{i} \times\left(n-n_{i}\right)$ matrix composed of the $i$ th row of blocks of $P$ with $P_{i, i}$ removed, $P_{i, i}$ is the $\left(n-n_{i}\right) \times n_{i}$ matrix composed of the $i$ th column of blocks of $P$ with $P_{i, i}$ removed, and $P_{i}$ is the $\left(n-n_{i}\right) \times\left(n-n_{i}\right)$ principal submatrix of $P$ with $i$ th row and $i$ th column of blocks removed. The $i$ th stochastic complement is the stochastic transition probability matrix of an irreducible MC of order $n_{i}$ obtained by observing the original process in the $i$ th NCD partition. The conditional steady state probability vector of the $i$ th NCD partition is $\pi_{i} /\left\|\pi_{i}\right\|_{1}$, and it may be computed by solving for the steady state vector of $\bar{P}_{i, i}$ (see [12] for details). However, each stochastic complement has an embedded matrix inversion which may require excessive computation.

Stochastic comparison is a technique by which both transient and steady state performance measures of a MC may be bounded. There are several applications of this technique in different areas of applied probability [17] and in practical problems of engineering [9,13,14]. The stochastic comparison of MCs is discussed in detail in $[10,11,20]$. The comparison of two MCs may be established by the comparison of their transient probability vectors at each time instant. Obviously, if steady states exist, stochastic comparison between their steady state probability vectors is also possible.

Sufficient conditions for the existence of stochastic comparison of two time-homogeneous MCs are given by the stochastic monotonicity and bounding properties of their one step transition probability matrices 
$[10,11]$. In [21], this idea is used to devise an algorithm that constructs an optimal st-monotone upperbounding MC. Later, this algorithm is used to compute stochastic bounds on performance measures that are defined on a totally ordered and reduced state space [1]. Performance measures may be defined as reward functions of the underlying MC. In [1], states having the same reward are aggregated, so the state space size of the bounding MC is considerably reduced. However, the given algorithm may provide loose bounds in the general case. If the state space reduction (i.e., aggregation) procedure takes into account the dynamics of the underlying system, it is possible to provide tight stochastic bounds. Another way to provide tight bounds is to consider specific matrix structures which are suitable to aggregation procedures.

In [22], a componentwise bounding algorithm for the state probability vector of NCD MCs is given. The two-level algorithm of Truffet in [22] uses aggregation and stochastic comparison with the strong stochastic (st) order. The algorithm is different from the bounded aggregation method discussed in $[3,16]$ in that a smaller number of linear systems of about the same order as those in [3] are solved at the cost of lower accuracy. The algorithm of Courtois and Semal in [3] uses polyhedra theory to compute the best possible bounds for a given NCD MC. To the best of our knowledge, the algorithm of Truffet has not been implemented and tested on any applications yet. Furthermore, its theoretical analysis lacks essential components. In this work, we present an improved and coherent version of the algorithm, and remedy the situation regarding analysis and implementation. We remark that even though the presentation of the algorithm in this paper is for computing the steady state probability vector of an NCD MC, it can also be used to carry out transient analysis with some modification. The bounding techniques in [3,22] both have tradeoffs. This work is not intended to be a comparative study between them, but it rather aims to develop a better understanding of stochastic comparison as a useful tool in performance analysis. In passing, we also remark that a continuoustime MC (CTMC) can be transformed through uniformization [19, p. 24] to a discrete-time MC. Hence, the algorithm presented in this paper may be used to compute bounds on the state probability vector of a CTMC.

In Section 2, we provide background on stochastic comparison. In Section 3, we introduce the improved algorithm and demonstrate the effect of the improvements on a small NCD MC. A thorough analysis of the new algorithm from the point of view of irreducibility appears in [15] and has not been included in the paper. In Section 4, we provide numerical results in sparse storage on a current application in mobile communications. Therein, we also discuss an application from the same research area which does not favor the algorithm. In Section 5, we conclude.

\section{Background on stochastic comparison}

There are different stochastic ordering relations and the most well known is the strong stochastic ordering (i.e., $\leqslant$ st). Intuitively speaking, two random variables $X$ and $Y$ which take values on a totally ordered space being comparable in the strong stochastic sense (i.e., $X \leqslant_{\mathrm{st}} Y$ ) means that it is less probable for $X$ to take larger values than $Y$ (see $[17,20]$ ).

First we give the definition of st-ordering used in this paper. For further information on the stochastic comparison method, we refer the reader to [20].

Definition 1. Let $X$ and $Y$ be random variables taking values on a totally ordered space. Then $X$ is said to be less than $Y$ in the strong stochastic sense, that is, $X \leqslant{ }_{\mathrm{st}} Y$ iff

$$
E[f(X)] \leqslant E[f(Y)]
$$

for all nondecreasing functions $f$ whenever the expectations exist.

Definition 2. Let $X$ and $Y$ be random variables taking values on the finite state space $\{1,2, \ldots, n\}$. Let $p$ and $q$ be probability vectors such that 


$$
p_{i}=\operatorname{Prob}(X=i) \quad \text { and } \quad q_{i}=\operatorname{Prob}(Y=i) \quad \text { for } i \in\{1,2, \ldots, n\} .
$$

Then $X$ is said to be less than $Y$ in the strong stochastic sense, that is, $X \leqslant_{\text {st }} Y$ iff

$$
\sum_{i=j}^{n} p_{i} \leqslant \sum_{i=j}^{n} q_{i} \text { for } j=n, n-1, \ldots, 1 .
$$

The comparison of MCs has been largely studied in [10,11,20]. We use the following definition (Definition 4.1.2 of [20, p. 59]) to compare MCs.

Definition 3. Let $\{X(t), t \in \mathscr{T}\}$ and $\{Y(t), t \in \mathscr{T}\}$ be two time-homogeneous MCs. Then $\{X(t), t \in \mathscr{T}\}$ is said to be less than $\{Y(t), t \in \mathscr{T}\}$ in the strong stochastic sense, that is, $\{X(t)\} \leqslant$ st $\{Y(t)\}$ iff

$$
X(t) \leqslant{ }_{\mathrm{st}} Y(t) \quad \forall t \in \mathscr{T} .
$$

It is shown in Theorem 3.4 of [11, p. 355] that monotonicity and comparability of the probability transition matrices of time-homogeneous MCs yield sufficient conditions for their stochastic comparison, which is summarized in:

Theorem 1. Let $P$ and $\widetilde{P}$ be stochastic matrices respectively characterizing time-homogeneous MCs $X(t)$ and $Y(t)$. Then $\{X(t), t \in \mathscr{T}\} \leqslant{ }_{\mathrm{st}}\{Y(t), t \in \mathscr{T}\}$ if

- $X(0) \leqslant{ }_{\mathrm{st}} Y(0)$,

- st-monotonicity of at least one of the probability transition matrices holds, that is,

either $P_{i, *} \leqslant \mathrm{st} P_{j, *} \quad$ or $\quad \widetilde{P}_{i, *} \leqslant{ }_{\mathrm{st}} \widetilde{P}_{j, *} \quad \forall i, j$ such that $i \leqslant j$,

- st-comparability of the transition matrices holds, that is,

$P_{i, *} \leqslant{ }_{\mathrm{st}} \widetilde{P}_{i, *} \quad \forall i$

Here $P_{i, *}$ refers to row $i$ of $P$.

\section{Componentwise bounding algorithm}

The componentwise bounding algorithm for the steady state vector of NCD MCs we present (see Algorithm 1) is based on the two-level algorithm in [22] that uses aggregation and stochastic comparison with the st-order.

\subsection{The improved algorithm}

Algorithm 1. Componentwise bounding algorithm for the steady state vector of NCD MCs:

0 . Find a (balanced) NCD partitioning of $P$ and symmetrically permute it to the form in Eq. (1). Let $\left\{\mathscr{S}_{1}, \mathscr{S}_{2}, \ldots, \mathscr{S}_{N}\right\}$ be the resulting state space partition.

1. For $i=1,2, \ldots, N$,

a. Choose a state from $\mathscr{S}_{i}$, say $f_{i}$, make it the last state and find the ordering of the remaining states in $\mathscr{S}_{i}$ with respect to $f_{i}$ by the heuristic algorithm in Step 3.a of $\left[5\right.$, p. 241]. Symmetrically permute $P_{i, i}$ according to the resulting ordering.

b. Compute the two stochastic matrices $\bar{S}_{i}$ and $\underline{S}_{i}$ of order $n_{i}$ corresponding to $P_{i, i}$ by Algorithms 2 and 3, respectively (see Remark 1). 
c. Compute the st-monotone upper-bounding matrix $\bar{Q}_{i}$ of order $n_{i}$ corresponding to $\bar{S}_{i}$ by Algorithm 5 and the st-monotone lower-bounding matrix $Q_{i}$ of order $n_{i}$ corresponding to $\underline{S}_{i}$ by Algorithm 6 .

d. Extract the irreducible submatrices of $\bar{Q}_{i}$ and $\underline{Q}_{i}$ and solve the corresponding systems of equations for their steady state vectors $\bar{\pi}_{i}^{\text {st }}$ and $\underline{\pi}_{i}^{\text {st }}$, respectively. Place zero steady state probabilities for transient states in each vector.

e. Compute the componentwise bounding vectors $\pi_{i}^{\text {sup }}$ and $\pi_{i}^{\text {inf }}$ on the conditional steady state probability vector corresponding to $\mathscr{S}_{i}$ from $\bar{\pi}_{i}^{\text {st }}$ and $\pi_{i}^{\text {st }}$ by Algorithm 7 .

2. a. Compute $U$ and $L$ of order $N$ using $\pi_{i}^{\text {sup }}$ and $\pi_{i}^{\text {inf }}, i \in\{1,2, \ldots, N\}$, by Algorithms 8 and 9 , respectively.

b. Compute the two stochastic matrices $\bar{S}$ and $\underline{S}$ of order $N$ corresponding to $L$ and $U$ by Algorithms 2 and 3 , respectively.

c. Compute the st-monotone upper-bounding matrix $\bar{Q}$ of order $N$ corresponding to $\bar{S}$ by Algorithm 5 and the st-monotone lower-bounding matrix $Q$ of order $N$ corresponding to $\underline{S}$ by Algorithm 6 .

d. Extract the irreducible submatrices of $\bar{Q}$ and $\bar{Q}$ and solve the corresponding systems of equations for their steady state vectors $\bar{\xi}^{\text {st }}$ and $\underline{\xi}^{\text {st }}$, respectively. Place zero steady state probabilities for transient states in each vector.

e. Compute the componentwise bounding vectors $\xi^{\text {sup }}$ and $\xi^{\text {inf }}$ on the steady state probability vector corresponding to $C$ from $\bar{\xi}^{\text {st }}$ and $\underline{\xi}^{\text {st }}$ by Algorithm 7 .

3. Compute the componentwise steady state probability upper- and lower-bounding vectors for $\mathscr{S}_{i}$ respectively as $\xi_{i}^{\text {sup }} \pi_{i}^{\text {sup }}$ and $\xi_{i}^{\text {inf }} \pi_{i}^{\text {inf }}, i \in\{1,2, \ldots, N\}$.

Remark 1. When Algorithms 2 and 3 are invoked for the substochastic matrices $P_{i, i}, L=P_{i, i}$ and $U=L+\Delta$, where $d=e-L e$ and $\Delta=\left[\begin{array}{llll}d & d & \cdots & d\end{array}\right]$.

Algorithm 2. Construction of stochastic matrix $\bar{S}$ corresponding to $L$ and $U$ of order $m$ :

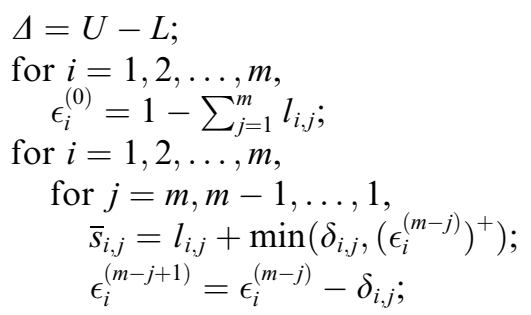

Algorithm 3. Construction of stochastic matrix $\underline{S}$ corresponding to $L$ and $U$ of order $m$ :

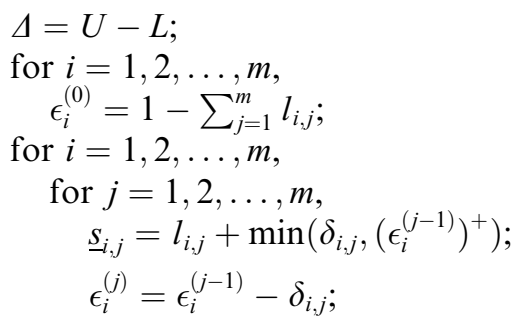

Algorithm 4. Construction of matrix $B$ (to be used in Algorithms 5 and 6) corresponding to stochastic matrix $S$ of order $m$ : 


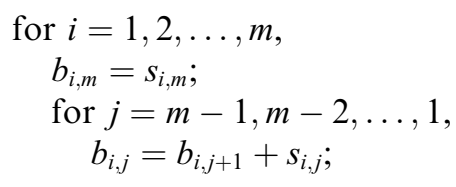

Algorithm 5. Construction of st-monotone upper-bounding matrix $\bar{Q}$ corresponding to stochastic matrix $\bar{S}$ of order $m$ :

Compute $B$ by Algorithm 4 for $\bar{S}$ of order $m$.

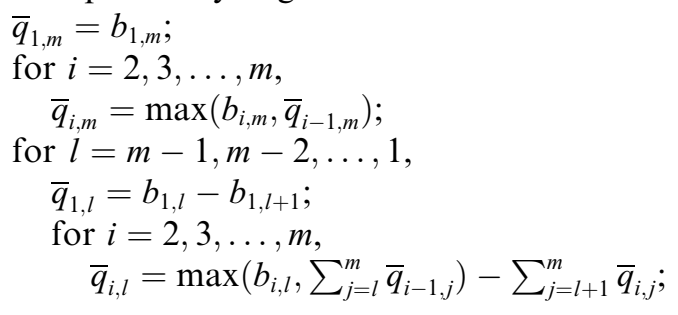

Algorithm 6. Construction of st-monotone lower-bounding matrix $\underline{Q}$ corresponding to stochastic matrix $\underline{S}$ of order $m$ :

Compute $B$ by Algorithm 4 for $\underline{S}$ of order $m$.

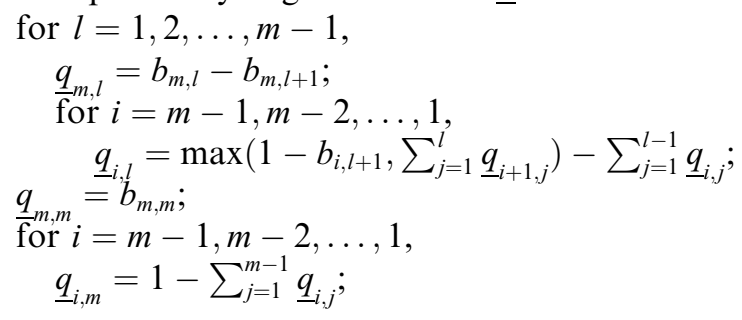

Algorithm 7. Computation of componentwise probability bounding vectors $v^{\text {sup }}$ and $v^{\text {inf }}$ given st upper- and lower-bounding probability vectors $\bar{v}^{\text {st }}$ and $\underline{v}^{\text {st }}$ of length $m$ :

$$
\begin{aligned}
& v_{m}^{\text {sup }}=\bar{v}_{m}^{\text {st }} ; \\
& v_{m}^{\text {inf }}=\underline{v}_{m}^{\text {st. }} ; \\
& \text { for } j=m-1, m-2, \ldots, 1, \\
& \quad v_{j}^{\text {sup }}=\sum_{k=j}^{m} \bar{v}_{k}^{\text {st }}-\sum_{k=j+1}^{m} \underline{v}_{k}^{\text {st }} ; \\
& \quad v_{j}^{\text {inf }}=\left(\sum_{k=j}^{m} \underline{v}_{k}^{\text {st }}-\sum_{k=j+1}^{m} \bar{v}_{k}^{\text {st }}\right)^{+} ;
\end{aligned}
$$

Algorithm 8. Computation of componentwise upper-bounding matrix $U$ for $C$ of order $N$ using $P$ and $\pi_{i}^{\text {sup }}$, $i \in\{1,2, \ldots, N\}$ :

$$
\begin{aligned}
& \text { for } i=1,2, \ldots, N, \\
& \quad \text { for } j=1,2, \ldots, N, \\
& \quad u_{i, j}=\min \left(\pi_{i}^{\text {sup }} P_{i, j} e, \max \left(P_{i, j} e\right)\right)
\end{aligned}
$$

Algorithm 9. Computation of componentwise lower-bounding matrix $L$ for $C$ of order $N$ using $P$ and $\pi_{i}^{\text {inf }}$, $i \in\{1,2, \ldots, N\}$ : 


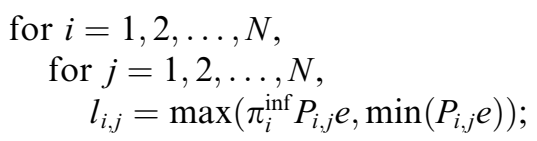

The preprocessing done in Step 0 of Algorithm 1 is self-descriptive. As suggested in Section 1, it is possible to use the algorithm in [4] to find NCD partitionings of $P$ given a user specified decomposability parameter. By a balanced partitioning, we mean one in which the $n_{i}, i \in\{1,2, \ldots, N\}$, in Eq. (1) do not differ significantly from each other. We argue why it is important to use balanced NCD partitionings after we analyze the complexity of Algorithm 1.

The ordering (i.e., numbering) of states in a MC affects the quality of bounds that may be obtained by the stochastic comparison approach [5] due to the conditions of st-monotonicity and st-comparability in Theorem 1. In order to obtain tighter probability bounds, Step 1.a of Algorithm 1 permutes one of the states within each NCD partition to be the last and orders the remaining states in the same partition using the heuristic given in [5, pp. 241-242]. The state to be permuted to the end of each NCD block is chosen as the state which has the largest self-transition probability among the states in the same NCD partition followed by a simple tie-breaking rule if needed. We do not reorder (aggregated) states in Step 2 of Algorithm 1 since the resulting matrices are highly diagonally dominant due to the NCD structure implying a small gain (if at all). Reordering of states is the first improvement over the algorithm in [22].

At the first level of Algorithm 1 (see Step 1), componentwise upper- and lower-bounds on the conditional steady state probability vector of each NCD partition are computed for the partitioning of $P$ in Eq. (1). This is achieved in Steps 1.b and 1.c (see Algorithms 2-6) by computing st-monotone upper- and lower-bounding matrices for each stochastic complement. In Step 1.b, two stochastic matrices corresponding to the particular NCD block are obtained using Algorithms 2 and 3. The former (latter) of these matrices is computed by adding the off-diagonal block probability mass to the incoming transitions of the last (first) state in the NCD partition thereby ensuring that the resulting stochastic matrix satisfies the st-comparability relation greater (less) than or equal to with the stochastic complement (see Section 1) of the NCD block. The two stochastic matrices obtained in this way for each NCD partition are input to Step 1.c. In Step 1.c, we use the st-monotone upper-bounding matrix construction algorithm in [1] as in [22] (see Algorithm 5), but devise and use a new st-monotone lower-bounding matrix construction algorithm (see Algorithm 6) whose optimality is proved in [15]. In [22], the st lower-bounding vector on the steady state distribution of a MC is computed by reversing the order of its states and running Algorithm 5 on the permuted MC. See [22, p. 847] for details. The new st-monotone lower-bounding matrix construction algorithm we present eliminates the need for a permutation vector to order the states of the input stochastic matrix in reverse.

Neither of the two st-monotone bounding matrices computed for each stochastic complement may be irreducible [1]. However, as we prove in [15], both of these matrices have one irreducible subset of states. This is also true for the st-monotone bounding matrices computed for the coupling matrix, $C$ (see Section 1), in Step 2. After identifying the transient states and removing them from each of the two st-monotone bounding matrices, the resulting irreducible stochastic matrices are solved for their steady state vectors in Step 1.d. This gives st upper- and lower-bounds on the conditional steady state probabilities of the particular NCD partition. In Step 1.e, componentwise bounds on the conditional steady state probability vector of the NCD partition are obtained from the st upper- and lower-bounding vectors using Algorithm 7. In [15], Algorithm 7 is shown to be better than Algorithm 10 used in [22]. This is the second improvement over the algorithm in [22].

At the second level (see Step 2), st-monotone upper- and lower-bounding matrices for $C$ corresponding to the partitioning of $P$ in Eq. (1) are computed using Algorithms 2-6, 8 and 9 in Steps 2.a-c. This is achieved by using the conditional steady state probability bounding vectors obtained for each NCD partition at the first level. Note that in Step 2.a, Algorithms 8 and 9 compute the matrices $U$ and $L$ which are respectively componentwise upper- and lower-bounding matrices for $C$. In Step 2.b, two stochastic matrices corresponding to $C$ are obtained by Algorithms 2 and 3 using $U$ and $L$. The former (latter) of these stochastic matrices is 
computed so as to satisfy the st-comparability relation greater (less) than or equal to with $C$. In Step 2.c, stmonotone bounding matrices corresponding to these two stochastic matrices are obtained. From the two stmonotone bounding matrices, two stochastic matrices corresponding to the irreducible subsets of states are extracted, and they are solved for their steady state vectors in Step 2.d. This gives st upper- and lower-bounds on the steady state probabilities of $C$. In Step 2.e, the st-bounding vectors obtained in the previous step are used by Algorithm 7 to compute componentwise bounds on the steady state probabilities of $C$.

Finally, in Step 3 of Algorithm 1, the componentwise upper-bounding (lower-bounding) vector on the conditional steady state distribution of each NCD partition is unconditioned by the corresponding upperbound (lower-bound) on the steady state probability of $C$. Hence, we obtain componentwise bounds on the global steady state distribution, $\pi$.

We remark that Steps 1.d and 2.d should omit the removal of transient states and replace the steady solution process with a transient solution procedure when performing transient analysis.

The theoretical analysis of the algorithm in [22] lacks essential components. There is no mention of the existence of a single irreducible subset of states in the st-monotone upper-bounding matrix computed by Algorithm 5. The possibility of computing a reducible st-monotone bounding matrix for a given irreducible Markov chain is stated in [1]. For the proposed methodology, the existence of a single irreducible subset of states must be proved for the matrices obtained by Algorithms 5 and 6 at both levels. Furthermore, the componentwise bounding algorithm that takes in st upper- and lower-bounding probability vectors (see Algorithm 7) is superior to its counterpart:

Algorithm 10. Computation of componentwise probability bounding vectors $w^{\text {sup }}$ and $w^{\text {inf }}$ as in [22] given st upper- and lower-bounding probability vectors $\bar{v}^{\text {st }}$ and $\underline{v}^{\text {st }}$ of length $m$ :

$$
\begin{aligned}
& w_{m}^{\text {sup }}=\bar{v}_{m}^{\text {st }} ; \\
& w_{m}^{w_{\text {inf }}}=\underline{v}_{m}^{\text {st }} ; \\
& \text { for } j=m-1, m-2, \ldots, 1, \\
& \quad w_{j}^{\text {sup }}=\min \left(1,\left(\sum_{k=j}^{m} \bar{v}_{k}^{\text {st }}-\sum_{k=j+1}^{m} w_{k}^{\text {inf }}\right)^{+}\right) ; \\
& \quad w_{j}^{\text {inf }}=\min \left(1,\left(\sum_{k=j}^{m} \underline{v}_{k}^{\text {st }}-\sum_{k=j+1}^{m} w_{k}^{\text {sup }}\right)^{+}\right) ;
\end{aligned}
$$

These theoretical issues are discussed in detail in [15]. Now we proceed with the complexity analysis of Algorithm 1.

\subsection{Complexity analysis}

We assume that $P$ has $n z$ nonzero elements distributed uniformly across the matrix. Note that $n z$ is considered to be $O(n)$ for sparse matrices. Hence, there will be roughly $k=n z / n$ nonzero elements per row/ column of $P$ and $k_{i}=n z \times n_{i} / n^{2}$ nonzero elements per row/column of $P_{i, i}$. The uniform assumption is neither an optimistic nor a pessimistic one. Now, assuming that Algorithm 1 is implemented in sparse storage and a direct method is employed in solving the linear systems in Steps 1.d and 2.d, its space complexity other than the storage set aside for $P$ is $\max \left\{O(n z), \max _{i}\left\{O\left(n_{i}^{2}\right), O\left(N^{2}\right)\right\}\right\}$ reals and integers from Steps 0, 1.b, 1.c, 2.b, and 2.c. Other steps contribute as lower order terms. As for the time complexity of the algorithm, we should account for floating-point comparisons and floating-point arithmetic operations separately. From Steps 0, 1.a-d, 2.a, and 2.d, we have $\max \left\{O\left(n k^{2}\right), \sum_{i=1}^{N} \max \left\{O\left(n_{i}^{2}\right), O\left(n_{i} k_{i}^{2}\right)\right\}\right.$, $\left.O(N n), O\left(N^{3}\right)\right\}$ floating-point comparisons. From Steps 1.d, 2.a, and 2.d, we have $\max \left\{\sum_{i=1}^{N} O\left(n_{i}^{3}\right), O(n z)\right.$, $\left.O(N n), O\left(N^{3}\right)\right\}$ floating-point arithmetic operations. Other steps contribute as lower order terms. Now it is evident why one should opt for balanced NCD partitionings (cf. Step 0).

Now, let us compare the time complexity of Algorithm 1 with that of iterative aggregation-disaggregation (IAD), a method devised to compute the steady state vector of NCD MCs using successive 
approximations [19, Chapter 6]. In order to make a fair comparison, let us assume that both methods use the same NCD partitioning and that there is space to factorize its diagonal blocks at the outset [6]. We make the same assumption regarding sparsity as in Algorithm 1 and conclude that there are roughly $\sum_{i=1}^{N} n_{i}\left(k-k_{i}\right)$ nonzero elements in the off-diagonal blocks of $P$. Each iteration of IAD consists of two steps. In the aggregation step, an approximate coupling matrix is formed and solved for its steady state vector. In order to expedite the computation of this matrix, row sums of each block in the NCD partitioning are computed and stored at the outset. In the disaggregation step, a block Gauss-Seidel (BGS) iteration is performed. This requires the solution of $N$ nonsingular linear systems whose coefficient matrices are the diagonal blocks factorized at the outset and whose right hand sides are computed using the nonzero elements in the off-diagonal blocks, the steady state vector of the approximate coupling matrix, and the previous steady state approximation. Hence, there are $\max \left\{\sum_{i=1}^{N} O\left(n_{i}^{3}\right), O(n z)\right\}$ floating-point arithmetic operations at the outset. The aggregation and disaggregation steps respectively cost $\max \left\{O\left(N^{3}\right), O(N n)\right\}$ and $\max \left\{O\left(\sum_{i=1}^{N} n_{i}\left(k-k_{i}\right)\right), \sum_{i=1}^{N} O\left(n_{i}^{2}\right)\right\}$ floating-point arithmetic operations per IAD iteration. The number of iterations taken by IAD to converge to a tolerance of $\epsilon$ is $O\left(\log \epsilon / \log \|F\|_{\infty}\right)$ since each iteration of IAD reduces the error in the approximate solution by a factor of degree of coupling (see Theorem 6.6 in [19, p. 340]). It is clear that Algorithm 1 runs faster than IAD as long as the coefficient matrix is dense and the degree of coupling is not exceedingly small.

Now we show how Algorithm 1 executes on a small example.

\subsection{An example}

Consider the $8 \times 8$ Courtois matrix [2]

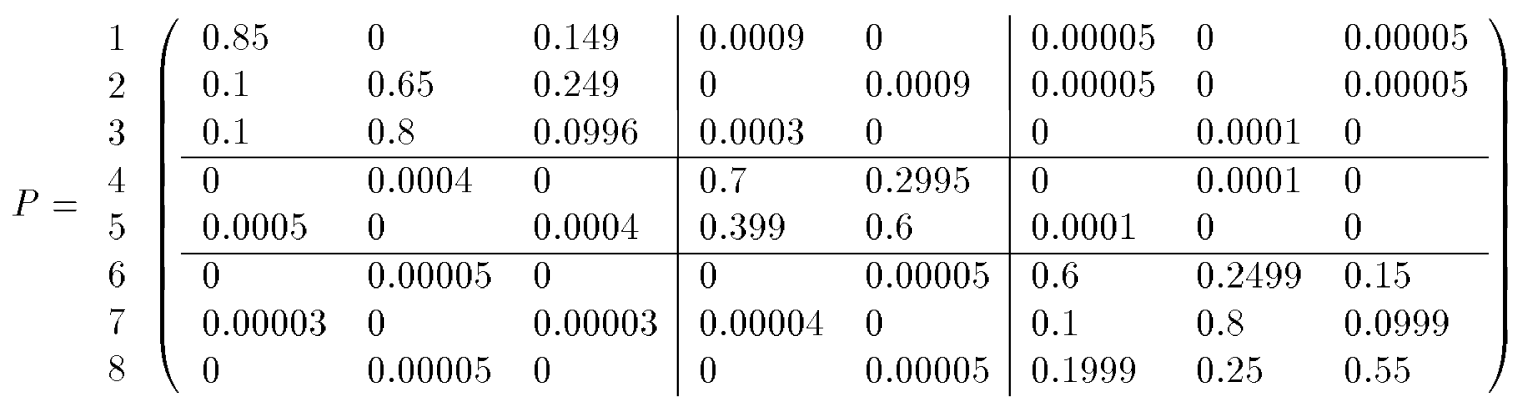

whose steady state vector is given by

$$
\pi=[0.089283,0.092758,0.040488,0.158533,0.118938,0.120385,0.277795,0.101819] .
$$

In Step 0, we choose a degree of decomposability [4] of 0.001 and obtain the state space partitioning $\left\{\mathscr{S}_{1}, \mathscr{S}_{2}, \mathscr{S}_{3}\right\}$, where $\mathscr{S}_{1}=\{1,2,3\}, \mathscr{S}_{2}=\{4,5\}$ and $\mathscr{S}_{3}=\{6,7,8\}$. This is an NCD partitioning with $\|F\|_{\infty}=0.001$. The NCD blocks are

$$
P_{1,1}=\left(\begin{array}{lll}
0.85 & 0 & 0.149 \\
0.1 & 0.65 & 0.249 \\
0.1 & 0.8 & 0.0996
\end{array}\right), \quad P_{2,2}=\left(\begin{array}{ll}
0.7 & 0.2995 \\
0.399 & 0.6
\end{array}\right), \quad P_{3,3}=\left(\begin{array}{lll}
0.6 & 0.2499 & 0.15 \\
0.1 & 0.8 & 0.0999 \\
0.1999 & 0.25 & 0.55
\end{array}\right) .
$$

In Step 1.a, states 1, 4, and 7 are chosen as the last states in their corresponding NCD blocks. Given these last states, the heuristic algorithm in [5] returns the orderings $(3,2,1),(5,4)$, and $(6,8,7)$ for NCD blocks 1,2 , and 3 , respectively. When the $P_{i, i}$ for $i \in\{1,2,3\}$ are symmetrically permuted with respect to these orderings, they become 


$$
\begin{aligned}
& P_{1,1}=\begin{array}{l}
3 \\
2
\end{array}\left(\begin{array}{lll}
0.0996 & 0.8 & 0.1 \\
0.249 & 0.65 & 0.1 \\
0.149 & 0 & 0.85
\end{array}\right), \quad P_{2,2}=\begin{array}{l}
5 \\
4
\end{array}\left(\begin{array}{ll}
0.6 & 0.399 \\
0.2995 & 0.7
\end{array}\right), \\
& P_{3,3}=\begin{array}{l}
6 \\
7 \\
7
\end{array}\left(\begin{array}{lll}
0.6 & 0.15 & 0.2499 \\
0.1999 & 0.55 & 0.25 \\
0.1 & 0.0999 & 0.8
\end{array}\right)
\end{aligned}
$$

Step 1.b computes two stochastic matrices for each of the (permuted) NCD blocks which are given by

$$
\begin{aligned}
& \bar{S}_{1}=\left(\begin{array}{lll}
0.0996 & 0.8 & 0.1004 \\
0.249 & 0.65 & 0.101 \\
0.149 & 0 & 0.851
\end{array}\right), \quad \underline{S}_{1}=\left(\begin{array}{lll}
0.1 & 0.8 & 0.1 \\
0.25 & 0.65 & 0.1 \\
0.15 & 0 & 0.85
\end{array}\right), \\
& \bar{S}_{2}=\left(\begin{array}{lll}
0.6 & 0.4 \\
0.2995 & 0.7005
\end{array}\right), \quad \underline{S}_{2}=\left(\begin{array}{ll}
0.601 & 0.399 \\
0.3 & 0.7
\end{array}\right), \\
& \bar{S}_{3}=\left(\begin{array}{lll}
0.6 & 0.15 & 0.25 \\
0.1999 & 0.55 & 0.2501 \\
0.1 & 0.0999 & 0.8001
\end{array}\right), \quad \underline{S}_{3}=\left(\begin{array}{lll}
0.6001 & 0.15 & 0.2499 \\
0.2 & 0.55 & 0.25 \\
0.1001 & 0.0999 & 0.8
\end{array}\right) .
\end{aligned}
$$

Using these stochastic matrices, Step 1.c computes st-monotone upper- and lower-bounding matrices for each NCD partition which are given by

$$
\begin{aligned}
& \bar{Q}_{1}=\left(\begin{array}{lll}
0.0996 & 0.8 & 0.1004 \\
0.0996 & 0.7994 & 0.101 \\
0.0996 & 0.0494 & 0.851
\end{array}\right), \quad \underline{Q}_{1}=\left(\begin{array}{lll}
0.25 & 0.65 & 0.1 \\
0.25 & 0.65 & 0.1 \\
0.15 & 0 & 0.85
\end{array}\right), \\
& \bar{Q}_{2}=\left(\begin{array}{ll}
0.6 & 0.4 \\
0.2995 & 0.7005
\end{array}\right), \quad \underline{Q}_{2}=\left(\begin{array}{ll}
0.601 & 0.399 \\
0.3 & 0.7
\end{array}\right), \\
& \bar{Q}_{3}=\left(\begin{array}{lll}
0.6 & 0.15 & 0.25 \\
0.1999 & 0.55 & 0.2501 \\
0.1 & 0.0999 & 0.8001
\end{array}\right), \quad \underline{Q}_{3}=\left(\begin{array}{lll}
0.6001 & 0.15 & 0.2499 \\
0.2 & 0.55 & 0.25 \\
0.1001 & 0.0999 & 0.8
\end{array}\right) .
\end{aligned}
$$

We remark that both st-monotone bounding matrices corresponding to each NCD partition in the Courtois example turn out to be irreducible. In other words, they do not have any transient states.

Step 1.d solves the st-monotone bounding matrices for their steady state vectors, which are given by

$$
\begin{aligned}
& \bar{\pi}_{1}^{\text {st }}=[0.099600,0.496639,0.403761], \quad \underline{\pi}_{1}^{\text {st }}=[0.210000,0.390000,0.400000], \\
& \bar{\pi}_{2}^{\text {st }}=[0.428163,0.571837], \quad \underline{\pi}_{2}^{\text {st }}=[0.429185,0.570815], \\
& \bar{\pi}_{3}^{\text {st }}=[0.240679,0.203597,0.555724], \quad \underline{\pi}_{3}^{\text {st }}=[0.240882,0.203616,0.555502] .
\end{aligned}
$$

Using these vectors, Step 1.e computes componentwise bounds on the conditional steady state probabilities of each NCD partition as

$$
\begin{aligned}
& \pi_{1}^{\text {sup }}=[0.210000,0.500400,0.403761], \quad \pi_{1}^{\text {inf }}=[0.099600,0.386239,0.400000], \\
& \pi_{2}^{\text {sup }}=[0.429185,0.571837], \quad \pi_{2}^{\text {inf }}=[0.428163,0.570815],
\end{aligned}
$$




$$
\pi_{3}^{\text {sup }}=[0.240882,0.203819,0.555724], \quad \pi_{3}^{\mathrm{inf}}=[0.240679,0.203393,0.555502] .
$$

Since Step 1 of Algorithm 1 is over, Step 2 starts executing. In Step 2.a, the matrices $U$ and $L$ of order 3 are computed using $\pi_{i}^{\text {sup }}$ and $\pi_{i}^{\text {inf }}, i \in\{1,2,3\}$, as

$$
U=\left(\begin{array}{lll}
0.999600 & 0.000877 & 0.000100 \\
0.000615 & 0.999500 & 0.000100 \\
0.000056 & 0.000044 & 0.999900
\end{array}\right), \quad L=\left(\begin{array}{lll}
0.999000 & 0.000737 & 0.000100 \\
0.000614 & 0.999000 & 0.000100 \\
0.000056 & 0.000044 & 0.999900
\end{array}\right) \text {. }
$$

In Step 2.b, the stochastic matrices $\bar{S}$ and $\underline{S}$ corresponding to $L$ and $U$ are computed as

$$
\bar{S}=\left(\begin{array}{lll}
0.999023 & 0.000877 & 0.000100 \\
0.000614 & 0.999286 & 0.000100 \\
0.000056 & 0.000044 & 0.999900
\end{array}\right), \quad \underline{S}=\left(\begin{array}{lll}
0.999163 & 0.000737 & 0.000100 \\
0.000615 & 0.999285 & 0.000100 \\
0.000056 & 0.000044 & 0.999900
\end{array}\right) \text {. }
$$

In Step 2.c, st-monotone upper- and lower-bounding matrices for $C$ are computed as

$$
\bar{Q}=\left(\begin{array}{lll}
0.999023 & 0.000877 & 0.000100 \\
0.000614 & 0.999286 & 0.000100 \\
0.000056 & 0.000044 & 0.999900
\end{array}\right), \quad \underline{Q}=\left(\begin{array}{lll}
0.999163 & 0.000737 & 0.000100 \\
0.000615 & 0.999285 & 0.000100 \\
0.000056 & 0.000044 & 0.999900
\end{array}\right) .
$$

These two bounding matrices are also irreducible. by

In Step 2.d, the st-monotone bounding matrices are solved for their steady state vectors, which are given

$$
\bar{\xi}^{\mathrm{st}}=[0.210388,0.289612,0.500000], \quad \underline{\xi}^{\mathrm{st}}=[0.230836,0.269164,0.500000] .
$$

Using these vectors, in Step 2.e componentwise bounds on the steady state probabilities of $C$ are computed as

$$
\xi^{\text {sup }}=[0.230836,0.289612,0.500000], \quad \xi^{\text {inf }}=[0.210388,0.269164,0.500000] .
$$
as

In Step 3, componentwise bounds on the steady state probabilities of each NCD partition are computed

$$
\begin{aligned}
& \xi_{1}^{\text {sup }} \pi_{1}^{\text {sup }}=[0.048476,0.115510,0.093203], \quad \xi_{1}^{\text {inf }} \pi_{1}^{\text {inf }}=[0.020955,0.081260,0.084155], \\
& \xi_{2}^{\text {sup }} \pi_{2}^{\text {sup }}=[0.124297,0.165611], \quad \xi_{2}^{\text {inf }} \pi_{2}^{\text {inf }}=[0.115246,0.153643], \\
& \xi_{3}^{\text {sup }} \pi_{3}^{\text {sup }}=[0.120441,0.101910,0.277862], \quad \xi_{3}^{\text {inf }} \pi_{3}^{\text {inf }}=[0.120339,0.101697,0.277751] .
\end{aligned}
$$

Finally, the componentwise bounding vectors are permuted back to their original orderings, and we obtain componentwise upper- and lower-bounding vectors on $\pi$ :

$$
\begin{aligned}
& \pi^{\text {sup }}=[0.093203,0.115510,0.048476,0.165611,0.124297,0.120441,0.277862,0.101910], \\
& \pi^{\text {inf }}=[0.084155,0.081260,0.020955,0.153643,0.115246,0.120339,0.277751,0.101697] .
\end{aligned}
$$

Compare the result of the improved algorithm with those of the following three cases:

(i) First improvement turned off (i.e., no reorderings used):

$$
\begin{aligned}
& \pi^{\text {sup }}=[0.093817,0.116272,0.048795,0.166606,0.125044,0.166694,0.309165,0.125083], \\
& \pi^{\text {inf }}=[0.083459,0.080588,0.020781,0.152774,0.114594,0.100000,0.208222,0.090835] .
\end{aligned}
$$


(ii) Second improvement turned off (i.e., Algorithm 10 used instead of Algorithm 7):

$$
\begin{aligned}
& \pi^{\text {sup }}=[0.093277,0.115602,0.049383,0.165698,0.124363,0.120552,0.277862,0.101910], \\
& \pi^{\text {inf }}=[0.084094,0.081201,0.020149,0.153538,0.115168,0.120228,0.277751,0.101697] .
\end{aligned}
$$

(iii) Both improvements turned off (i.e., basic algorithm):

$$
\begin{aligned}
\pi^{\text {sup }} & =[0.128242,0.124810,0.052378,0.168326,0.126335,0.200943,0.309165,0.125083], \\
\pi^{\text {inf }} & =[0.059553,0.079426,0.020482,0.143034,0.107289,0.065751,0.208222,0.090835] .
\end{aligned}
$$

After assessing the quality of the bounds, we conclude that the performance of Algorithm 1 on the Courtois example is extremely good, and it is superior to each of the three cases. However, the Courtois problem is small, and to have a better understanding of Algorithm 1, we must apply it to larger examples.

\section{Numerical results}

The implementation of the algorithms in Section 3 is done in compact sparse row (CSR) Harwell-Boeing format which requires for each coefficient matrix of order $m$ one real and one integer array of size $n z_{m}$ (i.e., number of nonzero elements in the coefficient matrix), one integer array of size $(m+1)$, and temporary workspace to accommodate fill-in during factorization. All code is written in Fortran/C and compiled in double precision with $g 77 / g c c$ on a SUN UltraSparcstation 10 with 128 MB of RAM running Solaris 2.6. The numerical experiments are timed using a $\mathrm{C}$ function that reports CPU time. Since the resulting NCD MCs are of moderate order (i.e., thousands of states) and sparsity (i.e., tens of nonzeros per row), we consider the direct solution method of Grassmann-Taksar-Heyman (GTH) [8] at each level of Algorithm 1. This method is a more robust version of Gaussian elimination (GE) in which arithmetic with only positive numbers is performed [7].

We compare the run-time of Algorithm 1 with that of GTH and IAD [19] which are both geared towards NCD MCs. In order to make a fair comparison, with IAD we use the same partitionings as in Algorithm 1. For all combinations of the integer parameters we considered, there is sufficient space to factorize in sparse format (that is, to apply sparse GE to) the diagonal blocks in IAD. Furthermore, we use BGS in the disaggregation step and employ a stopping tolerance of $10^{-15}$ on the infinity norm of the residual vector at each iteration. We remark that for each problem solved, the relative backward error in IAD turns out to be less than $10^{-16}$. See [6] for recent results on the computation of the steady state vector of Markov chains.

The first application that we consider arises in wireless asynchronous transfer mode (ATM) networks. In [23], a multiservices resource allocation policy (MRAP) is developed to integrate two types of service over time division multiple access (TDMA) frames in a mobile communication environment. These are the constant bit rate (CBR) service for two types of voice calls (i.e., handover calls from neighboring cells and new calls) and the available bit rate (ABR) service for data transfer. A single cell and single carrier frequency is modeled. However, the arrival process of data and the service process of calls we consider is quite general and subsumes the model in [23].

The TDMA frame is assumed to have $C$ slots. Handover requests have priority over new call arrivals and they respectively arrive with probabilities $p_{\mathrm{h}}$ and $p_{\mathrm{n}}$. Each voice call takes up a single slot of a TDMA frame but may span multiple TDMA frames whereas each data packet is served in a single slot of a single TDMA frame. When all the slots are full, incoming voice calls are rejected. The number of voice calls that may terminate in a given TDMA frame depends on the number of active calls and is modeled as a binomial process with parameter $p_{\mathrm{s}}$. The parameters of the model are $p_{\mathrm{h}}=C \times 10^{-5}, p_{\mathrm{n}}=C \times 5 \times 10^{-6}$, and $p_{\mathrm{s}}=C \times 5 \times 10^{-6}$. 
Data is queued in a FIFO buffer of size $B$ and has the least priority. The arrival of data packets is modeled as an on-off process. The process moves from the on state to the off state with probability $\alpha$ and from the off state to the on state with probability $\beta$. The load offered to the system is defined as $L=\beta /(\alpha+\beta)$. Assuming that the time interval between two consecutive on periods is $t$, the burstiness of such an on-off process is described by the square coefficient of variation, $S_{\mathrm{C}}=\operatorname{Var}(t) /[E(t)]^{2}$. In terms of $L$ and $S_{\mathrm{C}}$, we have $\beta=2 L(1-L) /\left(S_{\mathrm{C}}+1-L\right)$ and $\alpha=\beta(1-L) / L$. When the on-off process is in the on state, we assume that $i \in\{0,1,2,3\}$ data packets may arrive with probability $p_{\mathrm{d} i}$. The mean arrival rate of data packets in the on state is defined as $R=\sum_{i=1}^{3} i \times p_{\mathrm{d} i}$. Hence, the global mean arrival rate of data packets is given by $G=L \times R$. We set $\left(p_{d 0}, p_{\mathrm{d} 1}, p_{\mathrm{d} 2}, p_{\mathrm{d} 3}\right)=(0.4,0.3,0.2,0.1)$ implying $R=1.0$. When the buffer is full, any excess packet is dropped. We do not consider the arrival of multiple handovers or multiple new calls during a TDMA frame duration. Observe that there is orders of magnitude between the average interarrival time of voice calls and the average interarrival time of data packets, which makes this problem NCD. In fact, the smallest degree of coupling values we computed for this problem are in the order of $10^{-4}$.

The performance measures of interest are the blocking probability of voice calls and the dropping probability of data packets. If the underlying $\mathrm{MC}$ is represented by a three-component state descriptor $(a, b, c)$, where $a$ denotes the state of the data arrival process, $b$ denotes the number of data packets in the buffer and $c$ denotes the number of active voice calls, then the blocking probability of voice calls is given by

$$
\begin{aligned}
p_{\text {block }}= & {\left[\left(p_{\mathrm{n}}\left(1-p_{\mathrm{h}}\right)+\left(1-p_{\mathrm{n}}\right) p_{\mathrm{h}}+2 p_{\mathrm{n}} p_{\mathrm{h}}\right)\left(1-p_{\mathrm{s}}\right)^{C} \sum_{i=0}^{1} \sum_{j=0}^{B} \pi_{i, j, C}+p_{\mathrm{n}} p_{\mathrm{h}} C\left(1-p_{\mathrm{s}}\right)^{C-1} p_{\mathrm{s}} \sum_{i=0}^{1} \sum_{j=0}^{B} \pi_{i, j, C}\right.} \\
& \left.+p_{\mathrm{n}} p_{\mathrm{h}}\left(1-p_{\mathrm{s}}\right)^{C-1} \sum_{i=0}^{1} \sum_{j=0}^{B} \pi_{i, j, C-1}\right] /\left[p_{\mathrm{n}}\left(1-p_{\mathrm{h}}\right)+\left(1-p_{\mathrm{n}}\right) p_{\mathrm{h}}+2 p_{\mathrm{n}} p_{\mathrm{h}}\right],
\end{aligned}
$$

and the dropping probability of data packets is given by

$$
p_{\mathrm{drop}}=\left[\left(p_{\mathrm{d} 1}+2 p_{\mathrm{d} 2}+3 p_{\mathrm{d} 3}\right) \sum_{i=0}^{C} \pi_{1, B, i}+\left(p_{\mathrm{d} 2}+2 p_{\mathrm{d} 3}\right) \sum_{i=0}^{C} \pi_{1, B-1, i}+p_{\mathrm{d} 3} \sum_{i=0}^{C} \pi_{1, B-2, i}\right] /\left[p_{\mathrm{d} 1}+2 p_{\mathrm{d} 2}+3 p_{\mathrm{d} 3}\right] .
$$

We remark that the above formulae is defined on the product state space having $2(B+1)(C+1)$ states of which some are unreachable.

Using Algorithm 1, we tabulate the difference between upper and lower bounds on the blocking probability of voice calls and the dropping probability of data packets in the system when $(B, C)=(30,10)$ and $(B, C)=(60,30)$. We remark that it is the tightness of the upper and lower bounds and the time it takes to compute them that matter. Detailed results and plots appear in [15].

For the smaller problem in Table 1, the underlying MC that has 572 states and 20,198 nonzero elements takes 0.3 seconds to solve when $S_{\mathrm{C}}=1$ and 0.2 seconds to solve when $S_{\mathrm{C}}=10$ using Algorithm 1 . Steps 0 and 1.a take a total of about $0 \mathrm{~s}$. It takes 2.6 seconds to solve the same MC by GTH. It takes at least 1.5 seconds (5 iterations) to solve when $S_{\mathrm{C}}=1$ and at least 1.8 seconds (9 iterations) to solve when $S_{\mathrm{C}}=10$ using IAD. The NCD partitionings considered for $S_{\mathrm{C}}=1$ all have 11 blocks with orders between 42 and 62, and a degree of coupling $6 \times 10^{-4}$. The NCD partitionings considered for $S_{\mathrm{C}}=10$ all have 22 blocks with orders between 21 and 31, and degree of coupling values between $1 \times 10^{-1}$ (for $L=0.1$ ) and $2 \times 10^{-2}$ (for $L=0.9$ ).

For the larger problem in Table 2, the underlying MC that has 2,852 states and 217,778 nonzero elements takes 3.3 seconds (Step 0: $0.3 \mathrm{~s}$; Step 1.a: $0.3 \mathrm{~s}$ ) to solve when $S_{\mathrm{C}}=1$ and $2.5 \mathrm{~s}$ (Step 0: $0.3 \mathrm{~s}$; Step 1.a: $0.2 \mathrm{~s}$ ) to solve when $S_{\mathrm{C}}=10$ using Algorithm 1. It takes 260.0 seconds to solve the same MC by GTH. It takes at least 64.2 seconds ( 3 iterations) to solve when $S_{\mathrm{C}}=1$ and at least 75.4 seconds (4 iterations) to solve when $S_{\mathrm{C}}=10$ using IAD. The NCD partitionings considered for $S_{\mathrm{C}}=1$ all have 31 blocks with orders 
Table 1

Sample results of experiments with $(B, C)=(30,10)$

\begin{tabular}{lrll}
\hline$L$ & $S_{\mathrm{C}}$ & $p_{\text {block }}^{\text {sup }}-p_{\text {block }}^{\text {inf }}$ & $p_{\text {drop }}^{\text {sup }}-p_{\text {drop }}^{\text {inf }}$ \\
\hline 0.6 & 1 & $2 \times 10^{-3}$ & $2 \times 10^{-4}$ \\
& 10 & $2 \times 10^{-2}$ & $7 \times 10^{-4}$ \\
0.7 & 1 & $1 \times 10^{-3}$ & $1 \times 10^{-4}$ \\
& 10 & $1 \times 10^{-2}$ & $6 \times 10^{-4}$ \\
0.8 & 1 & $1 \times 10^{-3}$ & $1 \times 10^{-4}$ \\
& 10 & $8 \times 10^{-3}$ & $4 \times 10^{-4}$ \\
\hline
\end{tabular}

Table 2

Sample results of experiments with $(B, C)=(60,30)$

\begin{tabular}{lrll}
\hline$L$ & $S_{\mathrm{C}}$ & $p_{\text {block }}^{\text {sup }}-p_{\text {block }}^{\text {inf }}$ & $p_{\text {drop }}^{\text {sup }}-p_{\text {drop }}^{\text {inf }}$ \\
\hline 0.6 & 1 & $1 \times 10^{-18}$ & $3 \times 10^{-20}$ \\
& 10 & $2 \times 10^{-18}$ & $8 \times 10^{-20}$ \\
0.7 & 1 & $1 \times 10^{-18}$ & $3 \times 10^{-20}$ \\
& 10 & $2 \times 10^{-18}$ & $7 \times 10^{-20}$ \\
0.8 & 1 & $9 \times 10^{-19}$ & $3 \times 10^{-20}$ \\
& 10 & $1 \times 10^{-18}$ & $6 \times 10^{-20}$ \\
\hline
\end{tabular}

between 62 and 122, and a degree of coupling $5 \times 10^{-3}$. The NCD partitionings considered for $S_{\mathrm{C}}=10$ all have 62 blocks with orders between 31 and 61, and degree of coupling values between $2 \times 10^{-1}$ (for $L=0.1$ ) and $2 \times 10^{-2}$ (for $L=0.9$ ).

The time spent to compute bounds using Algorithm 1 is very promising compared to solving the NCD MCs using GTH or IAD. This is understandable since Algorithm 1 solves multiple smaller systems (i.e., two systems corresponding to each NCD block $i$ with order at most $n_{i}$ ) and two aggregated systems of order at most $N$ whereas GTH solves the global system of order $n$ and IAD performs a number of aggregationdisaggregation iterations. The bounds computed on $p_{\text {block }}$ and $p_{\text {drop }}$ using Algorithm 1 are highly acceptable; the bounds on $p_{\text {drop }}$ are tighter. Furthermore, the upper-bounds on $p_{\text {block }}$ computed by Algorithm 1 are mostly better than those computed by the basic algorithm (see [15]). Note that the $4(B+1)$ steady state probabilities used in computing $p_{\text {block }}$ comprise those $3(C+1)$ used in computing $p_{\text {drop. }}$. If we remove the unreachable states from the two formulae, there happens to be exactly $4(B+1)-2$ steady state probabilities that contribute to $p_{\text {block }}$ and 6 that contribute to $p_{\text {drop }}$. We remark that st-comparison is expected to provide better componentwise bounds for states placed towards the end of the underlying MC. When we compare the values of $4(B+1)-2$ with $N$, it is clear that not all states contributing to $p_{\text {block }}$ can be placed as such. This is an intuitive explanation for having tighter bounds on $p_{\text {drop }}$ compared to those on $p_{\text {block }}$. We believe this to be also the reason behind obtaining better bounds on $p_{\text {block }}$ with Algorithm 1 compared to the basic algorithm. Due to the reordering of states in Step 1.a of Algorithm 1, we expect more improvement on performance measures computed using a large number of states than those that depend on a few states. There are other factors that influence the quality of the computed bounds such as the NCD partitioning employed, the ordering chosen by our heuristic within each NCD block, and the irreducibility structure of the computed st-monotone matrices.

Regarding the time it takes to compute bounds with Algorithm 1, the most important factor is the nonzero structure of the underlying MC. Consider, for instance, the application in [18] which also happens to be in the area of communications. It introduces an $\mathrm{MMPP}\left|E_{k}\right| 1 \mid K$ continuous-time queueing model for a video source that is fed to an ATM multiplexer. When the number of stages in the Erlang (E) process approximating the deterministic service distribution is $k=5$, the buffer size of the multiplexer is $K=74$, the 
Markov Modulated Poisson Process (MMPP) modeling the arrival distribution has 8 states with a different arrival rate in each state, and the real parameters in the paper are used, we have a CTMC with $n=8 k(K+1)=3000$ states. The corresponding DTMC obtained through uniformization is highly NCD with a partitioning that has 8 blocks induced by the states of the MMPP. Each block is of order 375 and the degree of coupling is in the order of $10^{-5}$. However, in each of its rows, this NCD MC has a maximum of 10 nonzero elements 7 of which are in the off-diagonal blocks. The diagonal blocks which are in the form of a quasi-birth-and-death (QBD) process have a maximum of 3 nonzero elements and lend themselves to relatively sparse factorizations. Hence, IAD is able to compute the steady state vector rapidly, and Algorithm 1, which favors relatively dense NCD MCs, cannot be recommended in this case.

\section{Conclusion}

In this paper, we have given the first numerical results on an application with (an improved version of) a componentwise bounding algorithm for the state probability vector of nearly completely decomposable Markov chains. The given two-level algorithm uses aggregation and stochastic comparison with the strong stochastic (st) order. In order to improve accuracy, it employs reordering of the states and a better componentwise probability bounding algorithm given st upper- and lower-bounding probability vectors. A thorough analysis of the algorithm from the point of view of irreducibility has been done. The run-time of the algorithm is much better than that of GTH and iterative aggregation-disaggregation in sparse storage, and the quality of the computed bounds on steady state probabilities are highly acceptable for the chosen application. It is difficult to make strong generalizations, but it is our experience that this algorithm will be useful in relatively dense nearly completely decomposable Markov chains with highly unbalanced steady state probabilities, a small number of states accumulating a large probability mass, and a small degree of coupling. Future work should focus on utilizing the algorithm in computing transient performance measures.

\section{Acknowledgements}

We thank the anonymous referee for the remarks and suggestions, which led to an improved manuscript.

\section{References}

[1] O. Abu-Amsha, J.-M. Vincent, An algorithm to bound functionals of Markov chains with large state space, Rapport de recherche MAI n 25, IMAG, Grenoble, France, 1996.

[2] P.-J. Courtois, Decomposability: Queueing and Computer System Applications, Academic Press, New York, 1977.

[3] P.-J. Courtois, P. Semal, Bounds for the positive eigenvectors of nonnegative matrices and for their approximations by decomposition, Journal of the Association for Computer Machinery 31 (1984) 804-825.

[4] T. Dayar, Permuting Markov chains to nearly completely decomposable form, Technical Report BU-CEIS-9808, Department of Computer Engineering and Information Science, Bilkent University, Ankara, Turkey, 1998, Available from <http:// www. cs. bilkent. edu. tr/tech-reports/1998/ABSTRACTS. 1998. html>.

[5] T. Dayar, N. Pekergin, Stochastic comparison, reorderings, and nearly completely decomposable markov chains (NSMC'99), in: B. Plateau, W.J. Stewart, M. Silva (Eds.), Numerical Solution of Markov Chains, Prensas Universitarias de Zaragoza, Spain, 1999, pp. 228-246.

[6] T. Dayar, W.J. Stewart, Comparison of partitioning techniques for two-level iterative solvers on large, sparse Markov chains, SIAM Journal on Scientific Computing 21 (2000) 1691-1705.

[7] T. Dayar, W.J. Stewart, On the effects of using the Grassmann-Taksar-Heyman method in iterative aggregation-disaggregation, SIAM Journal on Scientific Computing 17 (1996) 287-303. 
[8] W.K. Grassmann, M.I. Taksar, D.P. Heyman, Regenerative analysis and steady state distributions for Markov chains, Operations Research 33 (1985) 1107-1116.

[9] J. Kamburowski, Stochastically minimizing the makespan in two-machine flow shops without blocking, European Journal of Operational Research 112 (1999) 304-309.

[10] J. Keilson, A. Kester, Monotone matrices and monotone Markov processes, Stochastic Processes and Their Applications 5 (1977) 231-241.

[11] W.A. Massey, Stochastic orderings for Markov processes on partially ordered spaces, Mathematics of Operations Research 12 (1987) 350-367.

[12] C.D. Meyer, Stochastic complementation, uncoupling Markov chains, and the theory of nearly reducible systems, SIAM Review 31 (1989) 240-272.

[13] N. Pekergin, Stochastic delay bounds on fair queueing algorithms, in: Proceedings of INFOCOM'99, New York, 1999, pp. 12121220.

[14] N. Pekergin, Stochastic performance bounds by state reduction, Performance Evaluation 36-37 (1999) 1-17.

[15] N. Pekergin, T. Dayar, D.N. Alparslan, Componentwise bounds for nearly completely decomposable Markov chains using stochastic comparison and reordering, Technical Report BU-CE-0202, Department of Computer Engineering, Bilkent University, Ankara, Turkey, 2002, Available from <http://www. cs. bilkent. edu. tr/tech-reports/2002/ABSTRACTS. 2002. html>.

[16] P. Semal, Analysis of large Markov models, bounding techniques and applications, Doctoral Thesis, Université Catholique de Louvain, Belgium, 1992.

[17] M. Shaked, J.G. Shantikumar, Stochastic Orders and Their Applications, Academic Press, San Diego, CA, 1994.

[18] P. Skelly, M. Schwartz, S. Dixit, A histogram-based model for video traffic behavior in an ATM multiplexer, IEEE/ACM Transactions on Networking 1 (1993) 446-459.

[19] W.J. Stewart, Introduction to the Numerical Solution of Markov Chains, Princeton University Press, Princeton, NJ, 1994.

[20] D. Stoyan, Comparison Methods for Queues and Other Stochastic Models, John Wiley \& Sons, Berlin, Germany, 1983.

[21] M. Tremolieres, J.-M. Vincent, B. Plateau, Determination of the optimal upper bound of a Markovian generator, Technical Report 106, LGI-IMAG, Grenoble, France, 1992.

[22] L. Truffet, Near complete decomposability: Bounding the error by stochastic comparison method, Advances in Applied Probability 29 (1997) 830-855.

[23] V. Vèque, J. Ben-Othman, MRAP: A multiservices resource allocation policy for wireless ATM network, Computer Networks and ISDN Systems 29 (1998) 2187-2200. 